\title{
SEMIEMPIRICAL ATOM-CENTERED DENSITY MATRIX PROPAGATION APPROACH TO TEMPERATURE-DEPENDENT VIBRATIONAL SPECTROSCOPY OF IRINOTECAN
}

\author{
BOJANA KOTESKA, MAJA SIMONOSKA CRCAREVSKA † MARIJA GLAVAS DODOV‡ JASMINA TONIC RIBARSKAફ \\ AND LJUPČO PEJOV
}

\begin{abstract}
In the present study, a molecular dynamics study of irinotecan molecule with the atom-centered density matrix propagation scheme was carried out at AM1 semiempirical level of theory, at series of different temperatures, ranging from 5 $\mathrm{K}$ to $300 \mathrm{~K}$. Molecular dynamics simulations were performed within the NVE ensemble, initially injecting (and redistributing among the nuclei) various amounts of nuclear kinetic energies to achieve the desired target temperatures. Subsequently to initial equilibration phase of $2 \mathrm{ps}$, productive simulations were carried out for $8 \mathrm{ps}$. The accuracy of simulations and the closeness of the generated trajectory to those at the Born-Oppenheimer surface were carefully followed and analyzed. To compute the temperature-dependent rovibrational density of states spectra, the velocity-velocity autocorrelation functions were computed and Fourier-transformed. Fourier-transformed dipole moment autocorrelation functions were, on the other hand, used to calculate the temperature-dependent infrared absorption cross section spectra. The finite-temperature spectra were compared to those computed by a static approach, i.e. by diagonalization of mass-weighted Hessian matrices at the minima located on the potential energy surfaces. Thermally-induced spectral changes were analyzed and discussed. The advantages of finite-temperature statistical physics simulations based on semiempirical Hamiltonian over the static semiempirical ones in the case of complex, physiologically active molecular systems relevant to intermolecular interactions between drugs and drug carriers were pointed out and discussed.
\end{abstract}

Key words: irinotecan, molecular dynamics, atom-centered density matrix propagation scheme, anharmonic vibrational frequencies, statistical physics simulations, theoretical spectroscopy

AMS subject classifications. 70F99, 82B30, 92E10

1. Introduction. Appropriate delivery of drugs and other physiologically active substances to the tissue in which they are expected to exert their activity is a fundamental issue in life sciences. We have actually witnessed a drastic paradigmatic shift in pharmaceutical sciences concerning the drug delivery issues with the advent of nanoscience [1]. The medical treatment of diseases, the very essence of drugs pharmacological activity as well as its distribution and metabolism critically depends on proper delivery. Often the delivery systems (the drug carriers) are designed such to enable a controlled release of the active ingredients as well. At the present state of the art within the field, various forms of drug delivery systems have evolved towards nanoparticles acting as encaging systems for the physiologically active components. To understand the physicochemical basis of the encaging phenomena, often a close collaboration between theory and experiment is crucial. In-depth studies of the molecular basis of the nanoparticle-drug intermolecular interactions (often being of noncovalent type) can even lead to a much more efficient design of novel carriers. In the present study, we tackle this issue focusing on a rather interesting and important hydrophilic drug irinotecan. Irinotecan is a rather important physiologically active substance, as it is used in the treatment of colon cancer, as well as in treatment of small cell lung cancer together with cisplatin. This hydrophilic drug has been recently incorporated into nanoparticle carriers composed by the poly lactic-co-glycolic acid copolymer (PLGA) and coadsorbed PEO-PPO-PEO (polyethylene oxide polypropylene polyethylene oxide) copolymer [2]. Among the other experimental techniques, Fourier transform infrared spectroscopy has been utilized to study the structural and dynamic changes of both guest and host molecules in the course of drug-nanopartice interaction upon encapsulation. The changes in experimentally measured spectral patterns upon encapsulations were shown to be rather subtle. Therefore, in order to get in-depth insights into the spectroscopic manifestations of the

*Faculty of Computer Science and Engineering, "Ss. Cyril and Methodious University", Rugjer Boskovikj 16, 1000 Skopje, Republic of Macedonia (bojana.koteska@finki.ukim.mk).

${ }^{\dagger}$ Institute of Pharmaceutical Technology, Center of Pharmaceutical Nanotechnology, Faculty of Pharmacy, "Ss. Cyril and Methodius University", Majka Tereza 47, 1000 Skopje, Republic of Macedonia(msimonoska@ff.ukim.edu.mk).

${ }^{\ddagger}$ Institute of Pharmaceutical Technology, Center of Pharmaceutical Nanotechnology, Faculty of Pharmacy, "Ss. Cyril and Methodius University", Majka Tereza 47, 1000 Skopje, Republic of Macedonia(magl@ff.ukim.edu.mk).

§Institute of Applied Chemistry and Pharmaceutical Analysis, Faculty of Pharmacy, "Ss. Cyril and Methodius University", Majka Tereza 47, 1000 Skopje, Republic of Macedonia(jato@ff.ukim.edu.mk).

ף Institute of Chemistry, Faculty of Science, "Ss. Cyril and Methodius University", P.O. Box 162, 1001 Skopje, Republic of Macedonia(ljupcop@pmf .ukim.mk). 
noncovalent interactions taking place between the incorporated drug molecule and the co-polymeric nanocarrier, substantial theoretical support is required. In the course of achieving this aim, we have recently undertaken a theoretical study of irinotecan molecule at several semiempirical levels of theory, as well as with a density functional theory (DFT) based approach [3]. We have shown in this study that theoretical treatment of this molecular system employing the semiempirical AM1 Hamiltonian is capable of reproducing most of its basic structural and spectroscopic properties computed at B3LYP/6-31G $(d, p)$ level of theory. However, as discussed in [3], static quantum mechanical calculations inherently refer to a system at $0 \mathrm{~K}$, while essentially all processes relevant to its physiological activity and incorporation into drug carrier systems occur at finite temperatures, quite above absolute zero. If one wants a reliable description of the physical phenomena in question, therefore, temperature-induced effects must be properly accounted for. To achieve this aim, in the present paper we study the temperature dependence of structure and vibrational spectroscopic properties of isolated irinotecan molecule employing molecular dynamics simulations based on the atom-centered density matrix propagation scheme [4] with a semiempirical AM1 Hamiltonian [5]. Using such methodological approach, sufficiently long simulations may be performed with a sufficiently accurate Hamiltonian for a proper description of the mentioned properties. This is a first step towards a development and implementation of rigorous theoretical approach aiming at an in-depth understanding of subtle structural and spectroscopic changes in the course of irinotecan incorporation (encapsulation) into drug nanocarrier systems.

\section{Computational details.}

2.1. Atom-centered density matrix propagation (ADMP) simulations. Semiempirical molecular dynamics simulations of free irinotecan molecule were carried out employing the atom-centered density matrix propagation (ADMP) scheme. This particular method belongs to the extended Lagrangian approaches to molecular dynamics, based on propagation of the density matrix, using Gaussian-type basis functions [4]. The extended Lagrangian of the studied system is written in the form:

$$
L=\frac{1}{2} \operatorname{Tr}\left(V^{T} M V\right)+\frac{1}{2} \mu \operatorname{Tr}(W W)-E(R, P)-\operatorname{Tr}[\Lambda(P P-P)]
$$

In (2.1), $M, R$ and $V$ are the nuclear masses, positions and velocities, respectively, while $P, W$ and $\mu$ denote the density matrix, density matrix velocity and the fictitious mass for the electronic degrees of freedom, correspondingly. $\Lambda$ is a Lagrangian multiplier matrix, and is here used to impose the constraints on the total number of electrons in the system and on the condition of idempotency of the density matrix.

Applying the principle of stationary action, one subsequently arrives at the Euler-Lagrange equations for density matrix propagation, which can be written in the form:

$$
\begin{gathered}
\mu \frac{d^{2} P}{d t^{2}}=-\left[\left.\frac{\partial E(R, P)}{\partial P}\right|_{R}+\Lambda P+P \Lambda-\Lambda\right] \\
M \frac{d^{2} R}{d t^{2}}=-\left.\frac{\partial E(R, P)}{\partial R}\right|_{P}
\end{gathered}
$$

For the purpose of the present study, equations (2.2) and (2.3) were integrated by the velocity Verlet algorithm. Within this algorithm, the density matrix propagation is given by:

$$
\begin{gathered}
P_{i+1}=P_{i}+W_{i} \Delta t-\frac{\Delta t^{2}}{2 \mu}\left[\left.\frac{\partial E\left(R_{i}, P_{i}\right)}{\partial P}\right|_{R}+\Lambda_{i} P_{i}+P_{i} \Lambda_{i}-\Lambda_{i}\right] \\
W_{i+1 / 2}=W_{i}-\frac{\Delta t}{2 \mu}\left[\left.\frac{\partial E\left(R_{i}, P_{i}\right)}{\partial P}\right|_{R}+\Lambda_{i} P_{i}+P_{i} \Lambda_{i}-\Lambda_{i}\right]=\frac{P_{i+1}-P_{i}}{\Delta t} \\
W_{i+1}=W_{i+1 / 2}-\frac{\Delta t}{2 \mu}\left[\left.\frac{\partial E\left(R_{i+1}, P_{i+1}\right)}{\partial P}\right|_{R}+\Lambda_{i+1} P_{i+1}+P_{i+1} \Lambda_{i+1}-\Lambda_{i+1}\right]
\end{gathered}
$$


Note that the extended Lagrangian molecular dynamics methodologies are especially well-suited for systems with very large number of the degrees of freedom, as the electronic subsystem is not treated by a full solution by e.g. a self-consistent field procedure; rather, it is propagated along with the nuclear degrees of freedom (which are, in turn, treated classically). This is achieved by an adjustment of the time scales of the mentioned motions (electronic and nuclear).

In the present study, as a starting point for the ADMP simulations, we have chosen the absolute minimum on the AM1 potential energy surface (PES) of the title molecule. This minimum has been obtained by our previous careful investigation of the potential energy landscapes of the title molecule employing a series of semiempirical Hamiltonians (AM1, PM3, PM6), as well as density functional levels of theory (e.g. B3LYP/6-31G(d,p)) [3]. The minimum has been located employing the Schlegel's gradient optimization algorithm [6]. Subsequently to the geometry optimization phase, harmonic vibrational analysis has been performed in order to compute the harmonic vibrational frequencies (at $0 \mathrm{~K}$ ) as well as to test the character of the located stationary point on the explored PES. Absence of imaginary frequencies (negative eigenvalues of the Hessian matrix) served as an indication that a true minimum on the PES has been reached.

Starting from the located minimum on the AM1 PES, semiempirical molecular dynamics simulations have been performed within the mentioned ADMP scheme. All ADMP simulations have been performed in the microcanonical $(N V E)$ ensemble. Various amounts of initial nuclear kinetic energies were initially injected to the system (and distributed among the atoms) in order to reach the finally desired temperatures. No thermostats were applied to maintain a constant temperature during each of the ADMP simulations. As shown below, such approach has led to acceptable temperature fluctuations throughout the simulation. Since we want to compute the spectroscopic properties of the title system within the dynamical approach, i.e. within the time correlation function approach, the dynamics of molecular system has to be sampled properly. Introducing a thermostat to maintain constant temperature, aside from allowing for much smaller temperature fluctuations, would however, severely distort the system's dynamics. Series of ADMP simulations were carried out at target temperatures of $5 \mathrm{~K}, 100 \mathrm{~K}, 150 \mathrm{~K}$ and at $300 \mathrm{~K}$. This temperature range was chosen to follow the temperature-evolution of the vibrational spectroscopic properties of the title molecule starting from a situation where quasi-harmonic behavior is expected, up to a situation which is often encountered in vibrational spectroscopic experiments under ambient conditions.

Upon initial velocity assignment, the system was allowed to equilibrate for 2 ps. Equilibration phase was followed by production (simulation) phase which was 8 ps long. To integrate the equations of motions, a time step of 0.2 fs was used for productive computations. The fictitious electron mass was set to 0.1 amu and the Cholesky basis for the orthonormal set was used.

Both the initial geometry optimizations with the semiempirical AM1 Hamiltonian and the subsequent ADMP simulations were performed with the Gaussian09 series of codes [7].

2.2. Time-correlation functions approach to spectroscopic properties. To compute the finitetemperature vibrational spectra of the studied irinotecan molecule from semiempirical molecular dynamics simulations, we relied on the time correlation functions approach, which is fundamentally based on the linear response formalism [8]. Within this approach, a particular autocorrelation function is computed from the collected data throughout the MD trajectory and this is sequentially Fourier-transformed to arrive at a spectrum of a given type. According to Wiener-Khintchine theorem [8], the autocorrelation function of a function of time $f(t)$ is given by:

$$
\langle f(\tau) f(t+\tau)\rangle_{\tau}=\frac{1}{2 \pi} \int\left|\int f(t) e^{-i \omega t} d t\right| e^{i \omega t} d \omega
$$

It follows from (2.7) that the autocorrelation of $\mathrm{f}(\mathrm{t})$ can be obtained by first taking the Fourier transform of $f(t)$ (which transforms it into the frequency domain), sequentially computing the square of its modulus and subsequently taking the inverse Fourier transform.

In the present study, we have used two types of autocorrelation functions to compute the vibrational spectra: autocorrelation function of the nuclear velocities (the velocity-velocity autocorrelation function) and the dipole moment autocorrelation function [9] [10]. 
The velocity-velocity autocorrelation function (VV-ACF) was computed from the data collected from the production (simulation) part of the ADMP trajectory as $[8,9,10]$ :

$$
\langle\vec{v}(t) \vec{v}(0)\rangle=\sum_{i} \sum_{j} \int_{0}^{T_{\text {lag }}} v_{i, j}\left(t^{\prime}\right) \cdot v_{i, j}\left(t^{\prime}+t\right) d t
$$

where $i$ ranges from 1 to the total number of atoms, while the index $j$ refers to the three principal Cartesian directions and ranges from 1 to 3 . Subsequently, VV-ACF was normalized with respect to the initial value $\langle\vec{v}(0) \vec{v}(0)\rangle$. From the normalized VV-ACF, the rovibrational density of states spectra, which are proportional to the kinetic energy spectra were computed by [8]:

$$
I_{v v}(\omega)=\lim _{T \rightarrow \infty} \int_{0}^{T} \frac{\langle\vec{v}(0) \vec{v}(0)\rangle}{\langle\vec{v}(0) \vec{v}(0)\rangle} e^{-i \omega t} d t
$$

In an analogous way, also the dipole moment autocorrelation function was computed from the ADMP simulation (production) phase and the infrared absorption cross-sections were computed by a subsequent Fourier transformation, i.e.:

$$
I_{\mu \mu}(\omega) \sim \lim _{T \rightarrow \infty} \int_{0}^{T} \frac{\langle\vec{\mu}(0) \vec{\mu}(0)\rangle}{\langle\vec{\mu}(0) \vec{\mu}(0)\rangle} e^{-i \omega t} d t
$$

Since in the case of both types of autocorrelation functions we computed the spectrum by Fourier transforming the time series obtained from simulations of finite length ADMP simulations, we have used the Blackmans window function to account for the fact that $T<\infty$ and cause the integrand to diminish at suitable $T$ values. Blackmans window function has the following form (in discrete notation) [11]:

$$
w(n)=0.42-0.5 \cdot \cos \left(\frac{2 \pi n}{N-1}\right)+0.08 \cdot \cos \left(\frac{4 \pi n}{N-1}\right) ; \quad 0 \leq n \leq N-1
$$

Fourier transformations for all purposes in the present study were performed with the fast Fourier transform (FFT) algorithm.

3. Results and discussion. The starting geometry of irinotecan molecule from which the ADMP simulations were started (corresponding to the minimum on the AM1 PES) is shown in Fig. 3.1.

As already implied in the methodology section, the atom-centered density matrix propagation scheme is an extended Lagrangian molecular dynamics method in which the electronic structure is accounted for by representing the electronic subsystem with a single-particle density matrix. This, in turn, is propagated simultaneously with the nuclear degrees of freedom (which are treated classically) by introducing the fictitious inertia tensor $\mu$ which practically results in an adjustment of the nuclear and electronic time scales.

In this manner, the resulting fictitious dynamics allows controllable oscillations around the Born-Oppenheimer surface. As in the ADMP scheme the self-consistence field (SCF) convergence is not achieved, one has to analyze carefully the errors in order to be certain of the accuracy of the dynamics as well as of its physical meaningfulness. In our present study, we have thoroughly analyzed the error by following the time-evolution of the adiabaticity index, as well as of the idempotency of the density matrix [9] [10]. We have also followed the time-dependence of the total angular momentum throughout the productive part of the simulation. Fig. 3.2 depicts the time-dependence of the adiabaticity index in the production phase of the simulation (subsequent to equilibration) at two working temperatures: $10 \mathrm{~K}$ and $150 \mathrm{~K}$.

As can be seen, the values of adiabaticity index indicate the stability of the simulations. This was also confirmed by checking out the idempotency of the density matrix, which was kept within the threshold value of $10^{-12}$. The total angular momentum value was conserved to $<10^{-13} \hbar$ as well. 


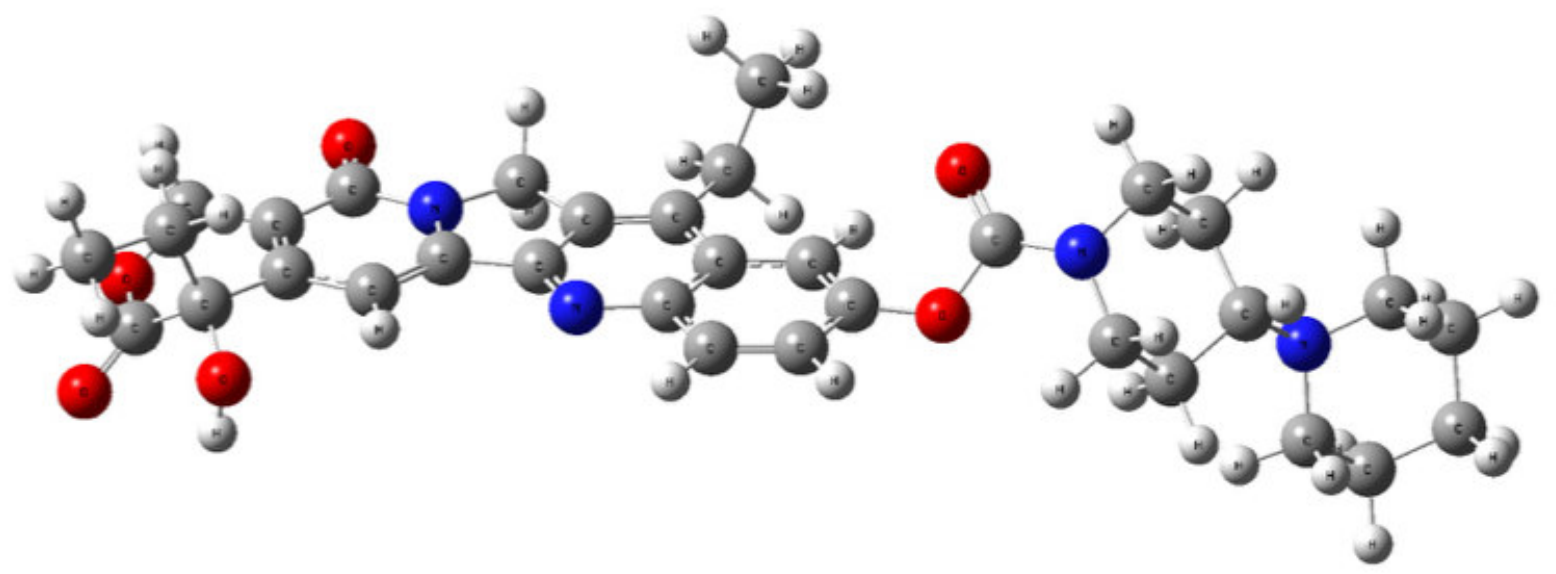

FIG. 3.1. The minimum located on the AM1 PES of irinotecan molecule (the starting geometry for the ADMP simulations).

The actually achieved average temperatures during the simulations compared to the target ones are given in Table 3.1. Temperature fluctuations around the target and average values presented in Table 3.1 were acceptable and in line with the statistical physics expectations for a dynamical simulation of molecular system with the current size. As mentioned in the Computational details section, however, temperature control has not been applied in the present study, since the main emphasis here is put on the computation of spectroscopic properties from dynamical simulations through the time correlation functions formalism. To do this properly, one needs to avoid the distortions of the dynamics which would be introduced by the imposed temperature control [9] [10].

TABLE 3.1

Target and actual temperatures achieved during the productive part of the ADMP simulation runs.

\begin{tabular}{|c|c|}
\hline$T_{\text {target }} / K$ & $T_{\text {sim. }} / K$ \\
\hline 5 & 5.0 \\
\hline 100 & 101.9 \\
\hline 150 & 152.7 \\
\hline 300 & 303.5 \\
\hline
\end{tabular}

Fig. 3.3 shows the kinetic energy spectra (i.e. the kinetic energy density of states spectra) obtained by Fourier transformation of the velocity-velocity autocorrelation function for the series of simulations carried out at the four different temperatures $(5,100,150$ and $300 \mathrm{~K})$. In this figure, the usually encountered frequency region in experimental studies (spanning from 500 to $4000 \mathrm{~cm}^{-1}$ ) is shown. In Figs. 3.4 and 3.5, on the other hand, the lower- (i.e. the fingerprint region) and higher-frequency ( $\mathrm{C}-\mathrm{H}$ as well as $\mathrm{O}-\mathrm{H}$ and $\mathrm{N}-\mathrm{H}$ stretching) regions of the kinetic energy spectra are shown.

Though the intensity pattern in the kinetic energy spectra is not directly comparable to the infrared spectrum, but rather to the deep inelastic neutron scattering spectrum, it still contains valuable information concerning the spacings between vibrational energy levels of different intramolecular modes. One can therefore follow the temperature evolution of the energy level differences, i.e. albeit in rather indirect way, the temperature evolution of the molecular conformational flexibility and intramolecular vibrational energy redistribution as well. Having these data for a free molecule of physiologically active substance is of essential importance for further studies and an in-depth understanding of its interaction with nanosized drug carriers.

Throughout the present study, we will use the kinetic energy spectra in parallel with those computed from the dipole autocorrelation function due to the following reasons. These type of spectra, as already implied before, contain information about the molecular rovibrational density of states, or, perhaps even more precisely, about the existence of energy level difference at particular frequency (wavenumber) value. Existence of a frequency difference, however, does not tell us anything about the particular mode that is involved. The intramolecular 


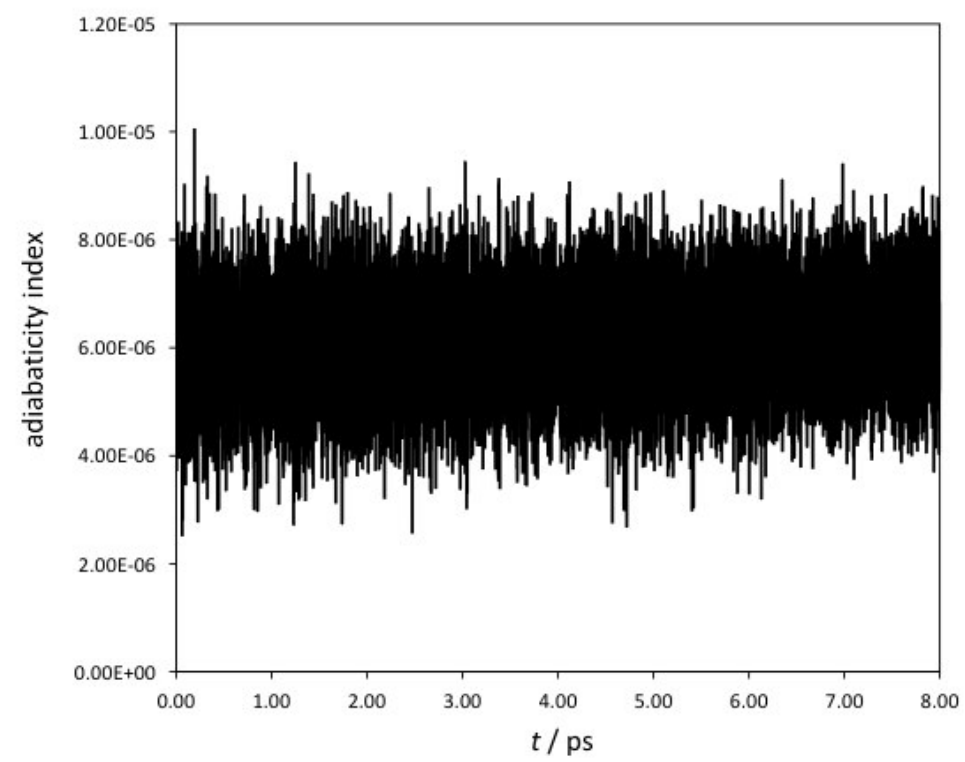

(a)

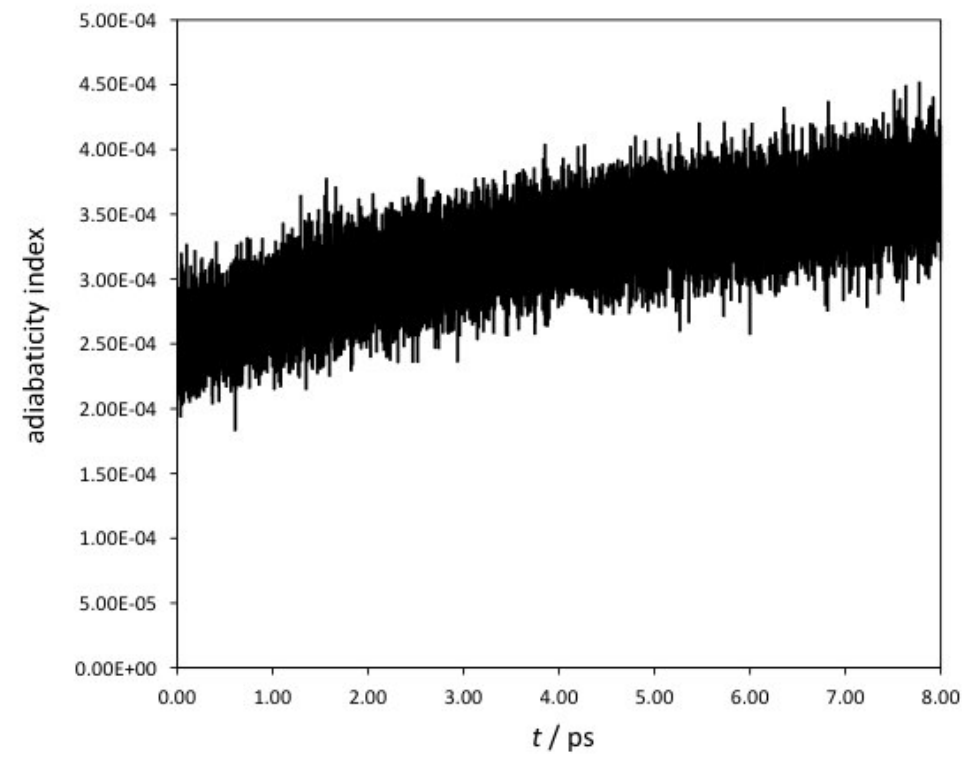

(b)

FIG. 3.2. Time-dependence of the adiabaticity index in the production phase of the simulation (sub-sequent to equilibration) at two working temperatures: $10 \mathrm{~K}(\mathrm{a})$ and $150 \mathrm{~K}(\mathrm{~b})$.

nuclear motions corresponding to that particular mode may not involve a change in the dipole moment which would be responsible for absorption of light quanta upon interaction with the incident radiation from infrared spectral region. If one therefore restricts the analysis solely on the basis of spectra obtained from the dipole moment auto-correlation function, the thermally-induced behavior of modes which are not infrared active would be left out.

Fig. 3.6, on the other hand, shows the spectra obtained by Fourier transformation of the dipole moment 


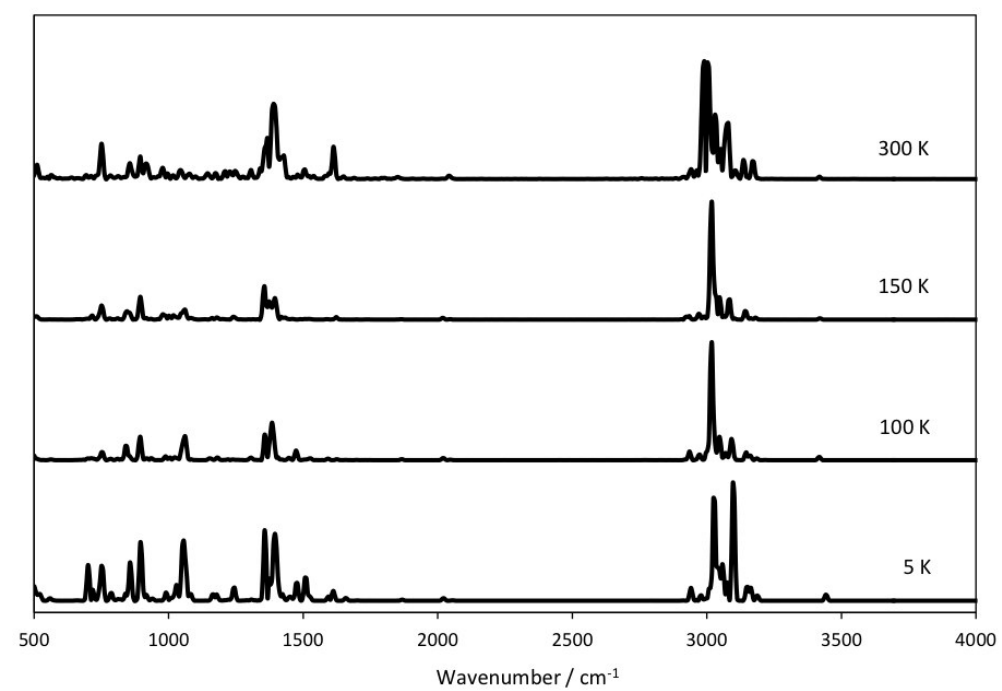

FIG. 3.3. The kinetic energy spectra (kinetic energy density of states spectra) obtained by Fourier transformation of the velocity-velocity autocorrelation function for the series of simulations carried out at the four different temperatures in the frequency region from 500 to $4000 \mathrm{~cm}^{-1}$.

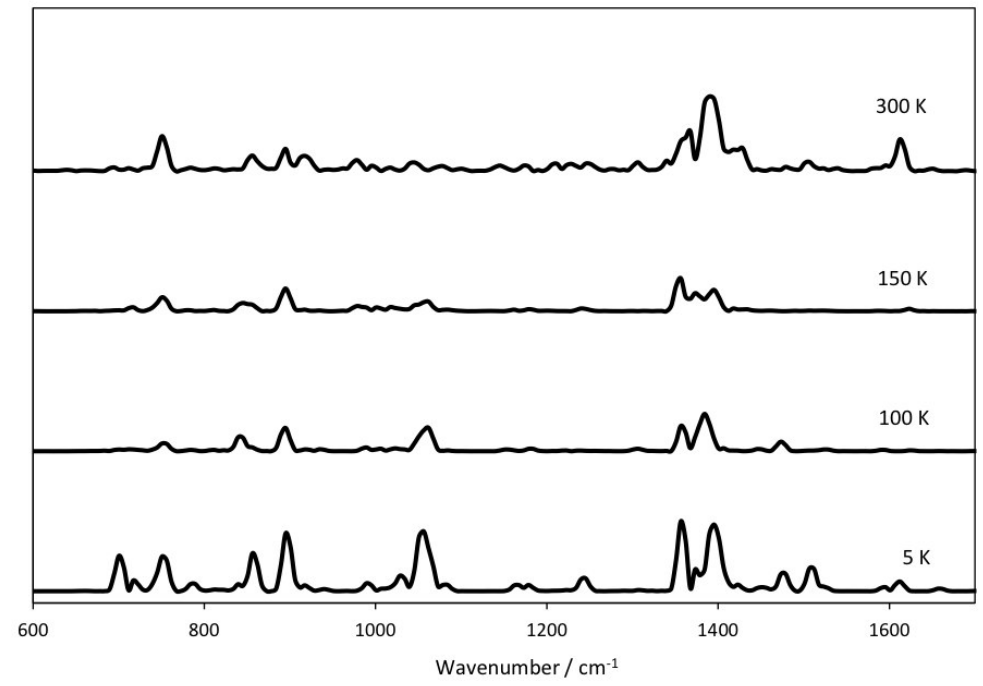

FIG. 3.4. The kinetic energy spectra (kinetic energy density of states spectra) obtained by Fourier transformation of the velocity-velocity autocorrelation function for the series of simulations carried out at the four different temperatures in the "fingerprint" frequency region from 600 to $1700 \mathrm{~cm}^{-1}$.

vector autocorrelation function for the series of simulations of free irinotecan molecule carried out at the four different temperatures $(5,100,150$ and $300 \mathrm{~K})$. Analogously as in Fig. 3.3, the usually encountered frequency region in experimental studies (spanning from 500 to $4000 \mathrm{~cm}^{-1}$ ) is shown here as well. These spectra should be directly comparable to the experimentally measured frequency dependencies of the infrared absorption crosssections obtained by experimental infrared spectroscopic techniques. Figs. 3.7 and 3.8 depict the lower- (i.e. the fingerprint region) and higher-frequency (C-H as well as $\mathrm{O}-\mathrm{H}$ and $\mathrm{N}-\mathrm{H}$ stretching) regions of the dipole moment autocorrelation spectra.

The intensity patterns shown in Figs. 3.6-3.8 should, therefore, be directly comparable to the temperature- 


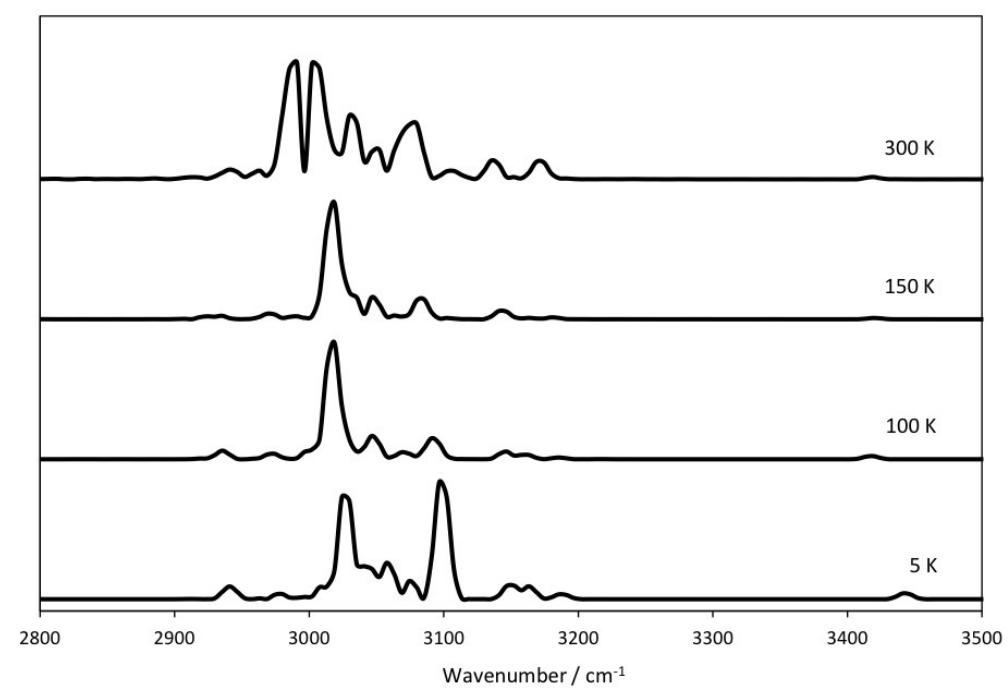

FIG. 3.5. The kinetic energy spectra (kinetic energy density of states spectra) obtained by Fourier transformation of the velocity-velocity autocorrelation function for the series of simulations carried out at the four different temperatures in the region of $\mathrm{C}-\mathrm{H}$ and $\mathrm{O}-\mathrm{H}$ stretching modes.

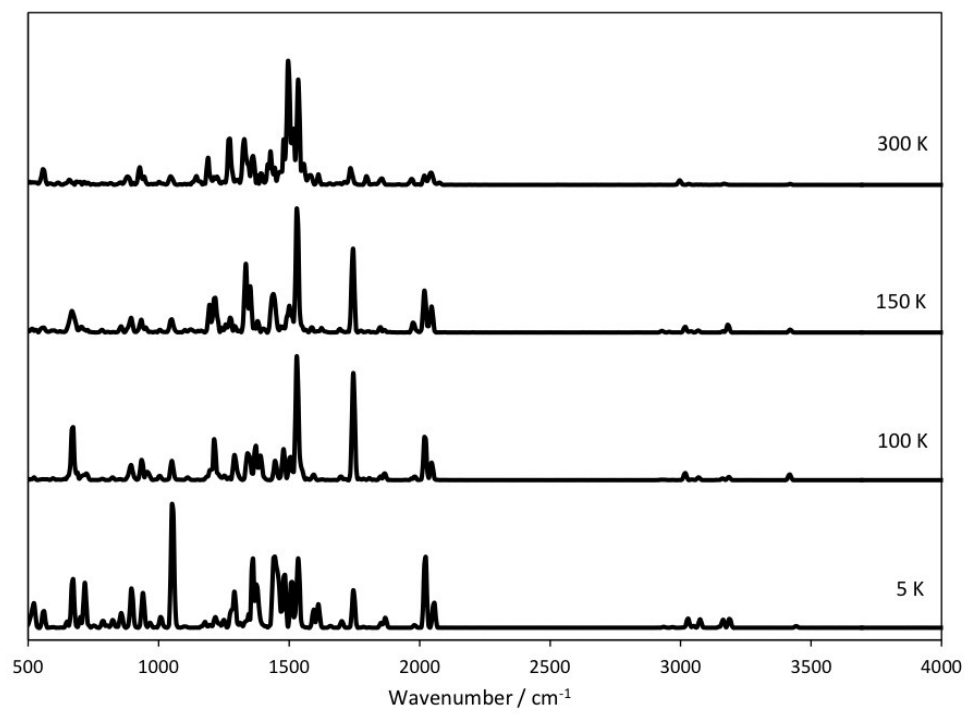

FIG. 3.6. The dipole moment autocorrelation spectra ( infrared absorption spectra) obtained by Fourier transformation of the dipole moment autocorrelation function for the series of simulations carried out at the four different temperatures in the frequency region from 500 to $4000 \mathrm{~cm}^{-1}$.

dependent infrared spectra of the studied species. Aside from the information related to the vibrational energy level spacings in the case of different intramolecular modes, from these spectra one can also directly follow the temperature evolution of the infrared absorption spectra, as well as the thermally-enhanced molecular conformational flexibility and intramolecular vibrational energy redistribution.

Fig. 3.9, finally shows the harmonic vibrational spectrum of free irinotecan molecule, computed in a "static" manner, i.e. by sequential geometry optimization (location of the minimum on the considered semiempirical PES) and computation and subsequent diagonalization of the mass-weighted Hessian matrix at this particular point on the PES. Note that such analysis, aside for the computation of the IR spectrum within the harmonic 


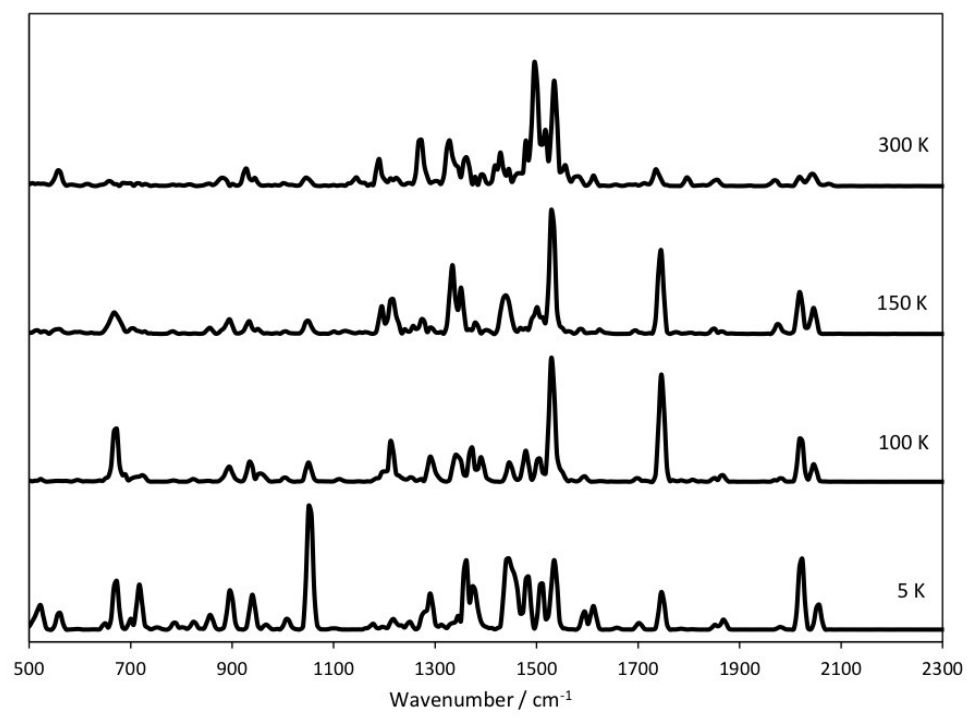

FIG. 3.7. The dipole moment autocorrelation spectra ( infrared absorption spectra) obtained by Fourier transformation of the dipole moment autocorrelation function for the series of simulations carried out at the four different temperatures in the "fingerprint" frequency region from 500 to $2300 \mathrm{~cm}^{-1}$.

approximation, has also served as a test of the character of the located stationary point(s) on the studied molecular PES. Absence of negative eigenvalues of the second-derivative matrices (i.e. absence of "imaginary frequencies") indicates that a true minimum on the considered PES has been located (instead of, e.g. a saddlepoint).

It is worth noting, however, that the harmonic vibrational spectrum depicted in Fig. 3.9 has been computed considering the minimum-energy structure of the studied molecular system at (implicitly assumed) temperature of $0 \mathrm{~K}$. It is, therefore, most directly (although not quantitatively) comparable to the dipole moment autocorrelation spectra calculated at $5 \mathrm{~K}$. The great advantage of the "dynamical spectra" is the fact that they inherently contain the influence of anharmonicity of the molecular vibrational modes, provided that the temperature is sufficiently high so that throughout the molecular dynamics simulation a sufficiently "wide" region of intramolecular motions is sampled (i.e. a sufficiently wide configurational space volume).

Both types of spectra computed from the ADMP simulations in the present study are based on the autocorrelation functions of averaged nuclear velocities or of the dipole moment vector. It is therefore worth recalling at this point that these are statistical quantities, which have been obtained as a statistical average from numerous different configurations spanned by the MD simulation.

The computed resultant spectrum should, therefore, correspond to a dynamically averaged picture of the studied molecular system. This, on the other hand, would result in an overall lowering of the intensity of the peaks (especially in the intermediate spectral region). At the same time, in the course of dynamical simulation, the immediate surroundings of each of the vibrational modes changes in a quite anisotropic manner. This, in consequence, leads to both broadening and flattening of particular spectral regions. Such observations are in line with previous results reported in the literature [9] [10].

The previously outlined theoretical results as well as the theoretical explanations behind such observations suggest that thermally-induced dynamical effects in the rovibrational density of states as well as in the infrared absorption cross-sections of individual molecular systems may lead to notables changes in comparison to the corresponding "static" properties (e.g. those computed for a particular stationary point on the studied PES or from a snapshot from a statistical physics simulation). This is especially important when one compares theoretical with experimental spectroscopic data, considering the fact that the later are often obtained at finite temperatures, much above the absolute 0 (which is the effective temperature at which "static" ab intio or semiempirical computations are often carried out). 


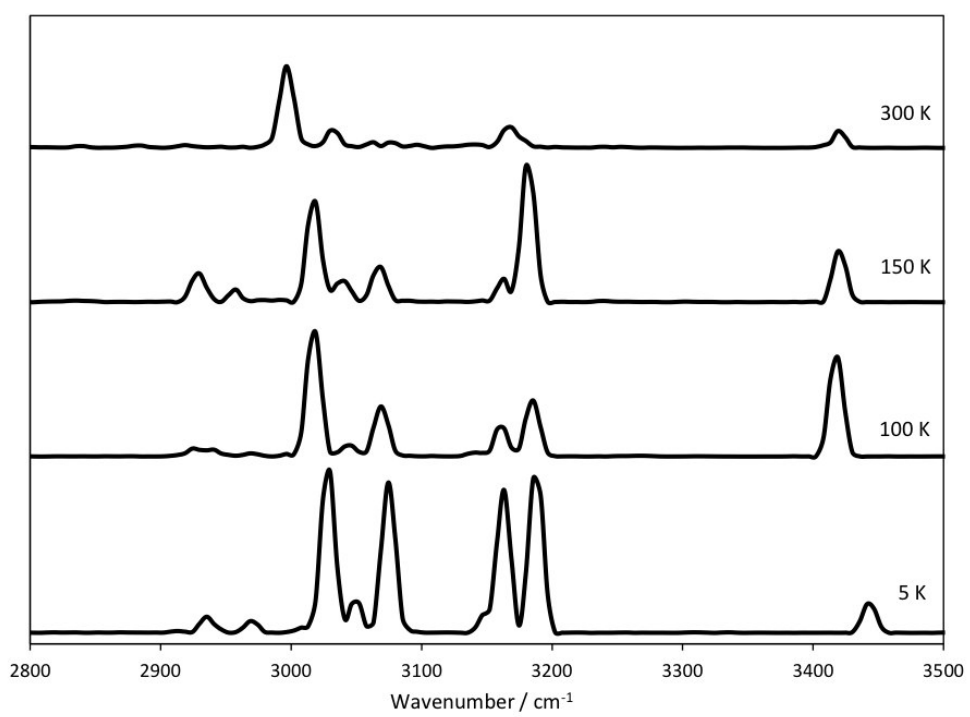

FIG. 3.8. The dipole moment autocorrelation spectra ( infrared absorption spectra) obtained by Fourier transformation of the dipole moment autocorrelation function for the series of simulations carried out at the four different temperatures in the region of $\mathrm{C}-\mathrm{H}$ and $\mathrm{O}-\mathrm{H}$ stretching modes.

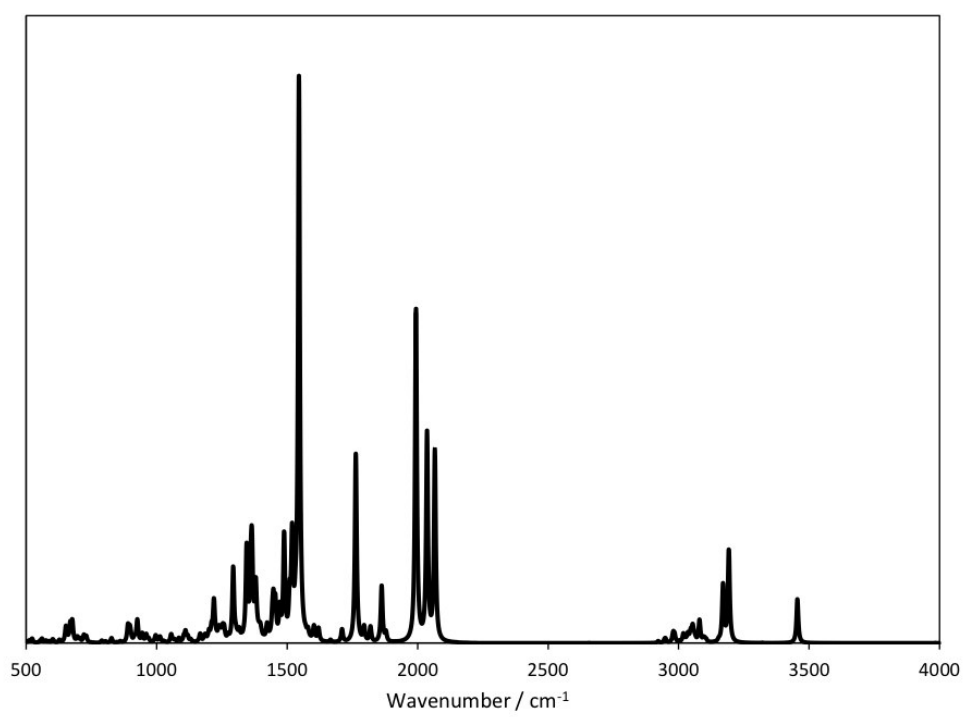

FIG. 3.9. The "static" harmonic vibrational spectrum computed by diagonalization of the mass-weighted Hessian matrix for the minimum located on the AM1 PES of the free irinotecan molecule.

In case when several conformers of a complex, large molecular system are close in energy, thermally-induced intramolecular motions and intramolecular vibrational energy redistributions may cause effective dynamical transitions between the corresponding wells on the molecular PES. The computed statistically averaged spectra from statistical physics simulations by the time correlation functions formalism therefore account for such dynamical effects and inherently account for the intramolecular conformational flexibility of the studied molecule. Such aspects are expected to be of essential importance especially when intermolecular interactions of noncovalent type are in questions. Such are, e.g. the interactions between drug molecules (as the presently studied irinotecan molecule) and drug carriers, cellular receptors, enzymes and other systems relevant in the biomolecular context. 
4. Summary and conclusions. In the present study, semiempirical molecular dynamics simulations of hydrophilic drug irinotecan were carried out employing the atom-centered density matrix propagation scheme at series of temperatures ranging from $5 \mathrm{~K}$ to $300 \mathrm{~K}$. From the computed molecular dynamics trajectories, various types of spectra were computed within the time correlation functions formalism. These included the rovibrational density of states spectra, which were computed from the velocity-velocity autocorrelation functions, as well as the infrared absorption cross section spectra, computed from the dipole moment autocorrelation functions. The thermally induced changes in the single-molecule spectroscopic properties were deduced and the reasons behind them were analyzed and discussed. This work is the basis for the development and implementation of an accurate and plausible statistical physics model for the drug nanocarrier intermolecular interactions and their spectroscopic manifestations.

Acknowledgments. This work was supported by the European Unions Horizon 2020 research and innovation programme, project Virtual Research Environment for Regional Interdisciplinary Collaboration in Southeast Europe and Eastern Mediterranean VI-SEEM [675121].

\section{REFERENCES}

[1] M.W. Tibbitt, J.E. Dahlman, And R. Langer. Emerging frontiers in drug delivery. Journal of the American Chemical Society, 138(3):704-717, 2016.

[2] M. Simonoska Crcarevska, N. Geskovski, S. Calis, S. Dimchevska, S. Kuzmanovska, G. Petruševski, M. KajdŽAnoska, S. Ugarkovic, And K. Goracinova. Definition of formulation design space, in vitro bioactivity and in vivo biodistribution for hydrophilic drug loaded plga/peo-ppo-peo nanoparticles using ofat experiments. European Journal of Pharmaceutical Sciences, 49(1):65-80, 2013.

[3] B. Koteska, A. Mishev, L. Pejov, M.S. Crcarevska, J.T. Ribarska, and M. G. Dodov. Computational Vibrational Spectroscopy of Hydrophilic Drug Irinotecan. In Proceedings of the Eighth International Conference on Advances in System Simulation - SIMUL), pages 11-16, 2016.

[4] H.B. Schlegel, J.M. Millam, S.S. Iyengar, G.A. Voth, A.D. Daniels, G.E. Scuseria, And M.J. Frisch. Ab initio molecular dynamics: Propagating the density matrix with gaussian orbitals. The Journal of Chemical Physics, 114(22):9758-9763, 2001.

[5] M.J.S. DewAR AND W. ThIEL. Ground states of molecules. 38. the mndo method. approximations and parameters. Journal of the American Chemical Society, 99(15):4899-4907, 1977.

[6] H.B. Schlegel. Optimization of equilibrium geometries and transition structures. Journal OF COMPUTATIONAL ChEMistry, $3(2): 214-218,1982$.

[7] M.J.E.A. Frisch, G.W. Trucks, Hs.B. Schlegel, G.E. Scuseria, M.A. Robb, J.R. Cheeseman, G. Scalmani, V. Barone, B. Mennucci, G.A. Petersson, et Al. Gaussian 09, Revision D. 01, 2009.

[8] M. Thomas, M. Brehm, R. Fligg, P. Vöhringer, and B. Kirchner. Computing vibrational spectra from ab initio molecular dynamics. Physical Chemistry Chemical Physics, 15(18):6608-6622, 2013.

[9] S.S. IYENGAR. Dynamical effects on vibrational and electronic spectra of hydroperoxyl radical water clusters. THE JouRNAL OF CHEMICAL PHYSICS, 123(8):084310, 2005.

[10] S.S. Iyengar, M.K. Petersen, T.J.F. Day, C.J. Burnham, V.E. Teige, and G.A. Voth. The properties of ion-water clusters. $i$. the protonated 21-water cluster. The Journal of CHEMICAL PHYSiCs, 123(8):084309, 2005.

[11] F.J HARRIS. On the use of windows for harmonic analysis with the discrete fourier transform. PROCEEDINGS OF THE IEEE, $66(1): 51-83,1978$.

Edited by: Aneta Karaivanova

Received: Dec 21, 2017

Accepted: Mar 12, 2018 